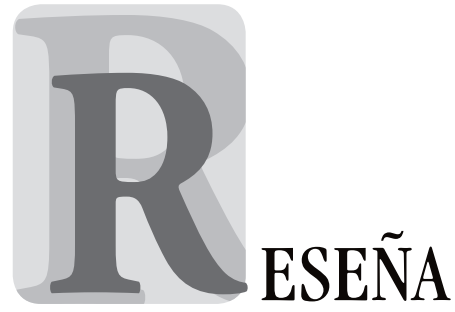

\title{
POLÍTICAS PÚBLICAS E INSTRUMENTOS PARA EL DESARROLLO DE LA CULTURA CIENTÍFICA EN AMÉRICA LATINA*
}

Desde tiempos remotos los saberes han transformado la manera de explorar lo desconocido y de adaptar lo que se tiene en el entorno para mejorar la calidad de vida. Esta exploración y adaptación están vinculadas por nexos, a veces imperceptibles pero irrompibles, por lo tanto, es indudable que el ser humano es afectado a diario, positiva o negativamente, por la tecnociencia, independientemente de sus afinidades o inclinaciones personales hacia los contenidos que esta les ofrece. Razón por la cual es importante implementar acciones políticas y operativas para que las personas del común hagan consciente el impacto sobre su vida, que tienen los desarrollos tecnocientíficos; que adquieran una postura activa como individuos que hacen parte de una sociedad, con criterios suficientes para exigir a través de decisiones personales y públicas que estos desarrollos realmente satisfagan sus necesidades básicas y cumplan las diferentes metas sociales deseables, en ámbitos como la justicia social, la educación con calidad, la participación democrática, mejores servicios culturales, servicios de salud y cuidado ambiental.

En la actualidad, el reto está en fomentar una cultura científica que vaya mucho más allá de la apropiación de lenguajes y teorías científicas, de forma tal que el individuo logre vincular los conocimientos adquiridos con su incidencia en la sociedad, incitándolo a apropiarse de temas públicos y a hacer uso de su derecho como ciudadano, en el ejercicio de la participación ciudadana informada, y en esta medida, tomar decisiones sólidamente fundamentadas.

* Fernández Polcuch, E.; Bello. A. y Massarani, L. (2016). Montevideo: LATU, UNESCO, RedPOP. 
Así las cosas, al participar de una sociedad del conocimiento, se reconoce el papel protagónico de las políticas públicas, las cuales aportan elementos invaluables a la construcción de una cultura científica enfocada al cultivo de la inteligencia del pueblo a través de vías formales y no formales, en las que se requiere de la vinculación de los agentes productores de información y de la utilización de los diversos medios de comunicación, para lograr este cometido.

En este sentido, el libro Politicas públicas e instrumentos para el desarrollo de la cultura cientifica en América Latina, resultado de un trabajo de investigación realizado por la Oficina Regional de Ciencias de la UNESCO para América Latina y el Caribe, con sede en Montevideo, y la Red de Popularización de la Ciencia y la Tecnología en América Latina y el Caribe (RedPOP), realizado con el proyecto del Conselho Nacional de Desenvolvimento Científico e Tecnológico $(\mathrm{CNPq}) /$ Brasil (Projeto $\mathrm{CNPq}$ 459257/2013-7), presenta un panorama de las iniciativas y perspectivas adoptadas por los países de esta región que permiten visualizar esos valores, ideas y programas que están plasmados en políticas públicas que aportan al desarrollo y modernización de los países.

Entre los aportes importantes del libro se encuentran las evidencias de iniciativas fuertes sobre cultura científica y de la renovada atención en el tema, plasmadas en políticas públicas donde se percibe como las decisiones en temas de ciencia y tecnología de los diferentes gobiernos denotan un incremento en la preocupación por la solución de necesidades sociales; también, en estas políticas se evidencia el fortalecimiento de una cultura científica en las poblaciones, lo que lleva a una visión más alentadora que la encontrada en registros de otros momentos históricos.

Por otra parte, se comienza con la identificación de la diversidad de denominaciones, conceptos y abordajes terminológicos para la concepción de una cultura científica cuyo trabajo fue fundamental para la investigación que les permitió la caracterización de las prácticas en cultura científica en los países de América Latina. Algunos términos encontrados en este estudio y que se consideran como sinónimos fueron: "popularización», «divulgación» $y$ "comunicación» de la ciencia. La «apropiación social de la ciencia» y el «desarrollo de la cultura científica» aparecen como conceptos estrechamente ligados, utilizados de manera creciente en los últimos ańos. El "periodismo científico", que de alguna manera es un término subordinado, también es utilizado, así como "difusión» y "promoción», que en realidad son algo ajeno al área específica. También se utiliza el término "comunicación pública de la ciencia», y su adopción es apoyada por numerosos expertos ( $\mathrm{p}$. 17). Es importante anotar que los autores para este informe adoptaron el término "desarrollo de una cultura científica», ya que no existe una definición internacionalmente reconocida que tenga un consenso académico acerca de su abordaje. Es de resaltar que, independiente del uso indistinto de estas denominaciones, el informe identifica unas intencionalidades y políticas importantes en el desarrollo de una cultura científica en los países de América Latina para acercarnos a sociedades del conocimiento sostenibles. En el abordaje de este tema, los autores profundizan en el concepto de cultura científica, en el papel de la cultura científica en las políticas, normativas e instituciones públicas de la región y en los diversos instrumentos de política de CTI.

En la segunda parte del informe presenta una innovadora herramienta de medición de las políticas de cultura científica, denominada «Huella de Políticas de Cultura Científica». La Huella provee una expresión gráfica del perfil que cada país adopta en sus políticas e instrumentos de política de fortalecimiento de la cultura científica, a partir de variables cuali-cuantitativos. La Huella permite, de esta manera, no solamente comparar entre países sino también detectar áreas de la política de cultura científica que pueden ser objeto de fortalecimiento (p. 31).

La última parte presenta el perfil detallado de todos los países objeto del presente estudio, tomando en consideración distintas variables que pueden influir en un estudio detallado de la cultura científica en el sector de Ciencia, Tecnología e Innovación (CTI).

Se analiza la composición del sistema nacional de CTI desde un punto de vista tanto de la normativa y prospectiva estructural, como de las políticas de 
CTI. Finalmente, se consideran los instrumentos con enfoque en el tema llevados a cabo por los países. Se brinda de esta manera un "perfil de país» o «Huella» del desarrollo de la cultura científica en la política de CTI. La "Huella» presenta una estructura flexible que puede ser ajustada en función de otros parámetros que quieran ser incorporados al análisis, en un futuro (p. 31). Esta valiosa información de la Huella de políticas de cultura científica de los países de la región es una radiografía de cómo los diferentes actores que interfieren en este proceso se han ido insertando tanto en lo institucional como en lo político, que sirven para la reflexión de cómo la gobernabilidad, legitimidad y normatividad juegan un papel importante a la hora de estructurar estas políticas.

Una de las conclusiones relevantes en este trabajo se refiere a que "América Latina y el Caribe están transitando un momento de auge en las políticas de Ciencia, Tecnología e Innovación (CTI), con un sustantivo aumento en la inversión en Investigación y Desarrollo Experimental ( $+\mathrm{D})$ en la última década. A su vez, la promoción de la "cultura científica" o "apropiación social del conocimiento" ha aumentado su prioridad en las agendas de los Organismos Nacionales de Ciencia y Tecnología (ONCYTs), los cuales han puesto en marcha diversos instrumentos en este sentido" (p. 40), lo que ratifica un panorama alentador en el que se nota un papel más activo y decidido, por ello, más intencional de los gobiernos.
Por último, este informe presenta un perfil de los países de la región: Argentina, Bolivia, Brasil, Colombia, Costa Rica, Ecuador, El Salvador, Guatemala, México, Paraguay, Perú y Uruguay. Para caracterizar los países lo hace desde tres perspectivas: dimensión normativa y prospectiva estructural: entidades ejecutoras de políticas y programas; instrumentos, programas y proyectos para el desarrollo de la cultura científica en el país; y la medición de la huella de la cultura científica en la política de CTI en cada uno de ellos, donde se constata que las tendencias en el desarrollo de la cultura científica son variadas entre los miembros de la comunidad andina, en la cual todos los países coinciden en un bajo nivel en cuanto a la intensidad de $\mathrm{I}+\mathrm{D}$, instrumentos de medición y marco legal.

En este panorama se identifica que «a pesar de los avances, sigue existiendo una situación fragmentaria o incipiente en relación a políticas públicas específicas y a la existencia de marcos legales para la popularización de la ciencia u otras estrategias del desarrollo de la cultura científica». Además, existe una cierta instabilidad y falta de continuidad con respecto a las políticas y estrategias en favor de la cultura científica, a ejemplo de lo que ocurre con la política en general en los países en América Latina. No obstante, se vislumbra que puede existir algo de conciencia desde los gobiernos, sin embargo, los esfuerzos son escasos $\mathrm{y}$ en algunos casos inexistentes.

Reseña elaborada por:

Lina Yanet Álvarez Estrada

Departamento de Biblioteca y Extensión Cultural Instituto Tecnológico Metropolitano -ITM

linaalvarez@itm.edu.co
Políticas públicas e instrumentos para el desarrollo de la cultura científica en América Latina 